\title{
GLOBAL BEHAVIOURS OF CIRCLES IN A COMPLEX HYPERBOLIC SPACE
}

\author{
By \\ Toshiaki AdACH* and Sadahiro MAEDA
}

\section{Introduction}

Let $M$ be a complete Riemannian manifold. $A$ curve $\gamma$ on $M$ parametrized by its arc length $s$ is called a circle if it satisfies the following equations

$$
\nabla_{s} X_{s}=k Y_{s}, \nabla_{s} Y_{s}=-k X_{s}, \quad \text { and } \quad X_{s}=\dot{\gamma}(s)
$$

for some positive constant $k$ and a field of unit vectors $Y_{s}$ along $\gamma$. Here $\nabla_{s}$ denotes the covariant differentiation along $\gamma$ with respect to the Riemannian connection $\nabla$ of $M$. The positive constant $k$ is called the curvature of $\gamma$. For given a positive $k$ and an orthonormal pair of vectors $u, v \in T_{x} M$ at a given point $x \in M$, we have a unique circle $\gamma$ defined for $-\infty<s<\infty$ such that $\gamma(0)=x, \dot{\gamma}(0)=u$ and $\left(\nabla_{s} \dot{\gamma}(s)\right)_{s=0}=k v$ (c.f. [7]). On a manifold of constant curvature the feature of circles with curvature $k$ is well-known. On a Euclidean space $\boldsymbol{R}^{n}$ they are circles (in usual sense of Euclidean geometry) of radius $1 / k$. On a sphere $S^{n}(c)$ of constant curvature $c$, they are small circles with prime period $2 \pi / \sqrt{k^{2}+c}$. In these cases all circles are closed. Here we call a circle $\gamma$ closed if there exists nonzero constant $s_{0}$ with $\gamma\left(s_{0}\right)=\gamma(0), X_{s_{0}}=X_{0}$ and $Y_{s_{0}}=Y_{0}$. The minimum positive $s_{0}$ satisfying these equalities is called the prime period of $\gamma$. On a real hyperbolic space $H^{n}(-c)$ of constant curvature $-c$, the feature of circles is different from these two cases (c.f. [5]). When the curvature $k$ of a circle is greater than $\sqrt{c}$ they are still closed with prime period $2 \pi / \sqrt{k^{2}-c}$. But when $k \leq \sqrt{c}$ they are unbounded. Similarly on a Hadamard surface it is known that circles are unbounded if their curvature is smaller than the square root of the absolute value of the upper bound of the curvature of the surface (see [2]).

In this paper we study global behaviours of circles on a complex hyperbolic space $\mathrm{CH}_{n}(-c)$ of holomorphic sectional curvature $-c$. For a circle $\gamma$ on a Kaehler manifold (with complex structure $J$ and with metric $\langle$,$\rangle ) we have an$

*The first author supported partially by The Sumitomo Foundation.

Recevied January 9, 1995. 
important invariant $\tau=\left\langle X_{s}, J Y_{s}\right\rangle$, which is called the complex torsion, defined by the associated vector fields $X_{s}$ and $Y_{s}$. This invariant does not depend on $s$;

$$
\begin{aligned}
\nabla_{s}\left\langle X_{s}, J Y_{s}\right\rangle & =\left\langle\nabla_{s} X_{s}, J Y_{s}\right\rangle+\left\langle X_{s}, J \nabla_{s} Y_{s}\right\rangle \\
& =k \cdot\left\langle Y_{s}, J Y_{s}\right\rangle-k \cdot\left\langle X_{s}, J X_{s}\right\rangle=0 .
\end{aligned}
$$

We find that two circles are congruent on a complex space form if and only if they have the same curvatures and the same complex torsions (c.f. Theorem 5.1 in [6]). Here we say that two circles $\gamma$ and $\sigma$ are congruent if there exists a holomorphic isometry $\varphi$ on a complex space form satisfying $\gamma=\varphi \circ \sigma$.

In the preceeding paper [3], we studied the feature of circles on a complex projective space $C P_{n}(c)$ of holomorphic sectional curvature $c$. We showed that there exist infinite many open circles: A circle on $C P_{n}(c)$ with curvature $k$ and complex torsion $\tau$ is closed if and only if (i) $\tau=0, \pm 1$ or (ii) a ratio of two solutions for the cubic equation $c \lambda^{3}-\left(4 k^{2}+c\right) \lambda+2 \sqrt{c} k \tau=0$ is rational. In this paper we show that the feature of circles on $\mathrm{CH}_{n}(-c)$ is more complicated.

THEOREM 1. Let $\gamma$ be a circle with curvature $k$ and with complex torsion $\tau$ on a complex hyperbolic space $\mathrm{CH}_{n}(-c)$ of holomorphic sectional curvature $-c$. For given $\tau$ we denote by $k(\tau)$ the unique positive solution for $k^{2} \tau^{2}-$ $(4 / 27)\left(k^{2}-1\right)^{3}=0$. Then the following hold:

(1) When $k \leq \sqrt{c} k(\tau) / 2, \gamma$ is a simple two-sides unbounded open curve. Here two-sides unbounded means that the sets $\{\gamma(s) \mid s \geq 0\}$ and $\{\gamma(s) \mid s \leq 0\}$ are unbounded.

(2) When $k>\sqrt{c k}(\tau) / 2$ and $\tau=0, \gamma$ is a simple closed curve with prime period $4 \pi / \sqrt{4 k^{2}-c}$.

(3) When $k>\sqrt{c k}(\tau) / 2$ and $\tau=1$ or $-1, \gamma$ is a simple closed curve with prime period $2 \pi / \sqrt{k^{2}-c}$.

(4) When $k>\sqrt{c} k(\tau) / 2$ and $\tau \neq 0, \pm 1$, we denote by $a, b$ and $d(a<b<d)$ the nonzero real solutions for

$$
c \lambda^{3}-\left(4 k^{2}-c\right) \lambda-2 \sqrt{c} k \tau=0 .
$$

Then we find the following:

(i) If one of the three ratios $a / b, b / d$ and $d / a$ is rational, $\gamma$ is a simple closed curve. Moreover, the prime period of $\gamma$ is the least common multiple of $4 \pi / \sqrt{c}(b-a)$ and $4 \pi / \sqrt{c}(d-a)$.

(ii) If each of the three ratios $a / b, b / d$ and $d / a$ is irrational, $\gamma$ is a simple bounded open curve. 
For a Hadamard manifold we have an important notion of the ideal boundary. Real hyperbolic space and complex hyperbolic space are typical examples of Hadamard manifold. With the ideal boundary we compactify $\mathrm{CH}_{n}(-c)$ and denote by $\overline{\boldsymbol{C} H_{n}(-c)}$. For a two-sides unbounded curve $\gamma$ we denote by $\gamma(\infty)$ (resp. $\gamma(-\infty)$ ) if the limit $\lim _{s \rightarrow \infty} \gamma(s)$ (resp. $\lim _{s \rightarrow-\infty} \gamma(s)$ ). exists in $\overline{\mathrm{CH}}(-c)$. When these points on the ideal boundary exist, we say that $\gamma$ has points at infinity. From this point of view the Comtet's result is rewritten as follows: In a real hyperbolic space $H^{n}(-c)$, every circle $\gamma$ with curvature $k \leq \sqrt{c}$ has points at infinity. When $k=\sqrt{c}$ they coincide; $\gamma(\infty)=\gamma(-\infty)$, and when $k<\sqrt{c}$ they are distinct; $\gamma(\infty) \neq \gamma(-\infty)$. For circles on a complex hyperbolic space we can show the following.

THEOREM 2. Let $\gamma$ denote a circle with curvature $k$ and complex torsion $\tau$ on $\mathrm{CH}_{\boldsymbol{n}}(-c)$. Then the following hold:

(1) If $k=\sqrt{c} k(\tau) / 2$, then $\gamma$ is horocyclic. Here horocyclic means that $\gamma(\infty)=\gamma(-\infty)$ and every geodesic $\rho$ with $\rho(\infty)=\gamma(\infty)$ crosses $\gamma$ orthogonally.

(2) If $k<\sqrt{c k}(\tau) / 2$, then $\gamma$ has two distinct points at infinity.

In section 1 we show by using the structure of the $S^{1}$-fiber bundle $M$ over $\mathrm{CH}_{n}(-c)$ that horizontal lifts of circles on $\mathrm{CH}_{n}(-c)$ into $\mathrm{M}$ are helixes of order 2, 3 or 5. This gives us explicit expressions of circles in $C_{n}(-c)$. By using of the explicit expressions of circles, we can investigate global behaviour of circles in $\mathrm{CH}_{n}(-c)$. In section 2 we use another expression of complex hyperbolic space, which is regarded as an open unit ball in $C^{n}$, and show Theorem 2. These generalize some results in [1].

\section{Open circles and closed circles}

Let $\gamma$ be a circle on a Riemannian manifold $(M, g)$ with curvature $k$. When we change the metric homothetically $g \rightarrow m^{2} \cdot g$ for some positive constant $m$, the curve $\sigma(s)=\gamma(s / m)$ is a circle on $\left(M, m^{2} \cdot g\right)$ with curvature $k / m$. Since we can obtain $\mathrm{CH}_{n}(-c)$ by changing the metric on $\mathrm{CH}_{n}(-4)$ homothetically; $g \rightarrow(4 / c) g$, and the complex torsion does not change in this operation, we may just treat the case $c=4$. Here, in general we note that on a Riemannian manifold $(M, g)$ under the operation $g \rightarrow m^{2} g$, the prime period of a closed circle changes to $\mathrm{m}$-times of the original prime period.

We shall start with giving some fundamental notations on a complex hyperbolic space of holomorphic sectional curvature -4 . We denote by $\ll, \gg$ the 
Hermitian form on $\boldsymbol{C}^{n+1}$ given by

$$
\left\langle z, w 》=-z_{0} \bar{w}_{0}+\sum_{j=1}^{n} z_{j} \bar{w}_{j}\right.
$$

for $z=\left(z_{0}, z_{1}, \ldots, z_{n}\right)$ and $w=\left(w_{0}, w_{1}, \ldots, w_{n}\right) \in C^{n+1}$. We define an indefinite metric $\langle$,$\rangle on C^{n+1}$ by $\left.\langle\rangle=,\operatorname{Re} \ll,\right\rangle$. Let $M$ be the real hypersurface with indefinite metric $\langle$,$\rangle in C^{n+1}$ defined by $\langle z, z\rangle=-1$. The group $S^{1}=\left\{e^{i \theta}\right\}$ acts freely on $M$ by $z \rightarrow e^{i \theta} z$. Hence we can consider a base manifold $M^{\prime}$ of the principal fiber bundle $\pi: M \rightarrow M^{\prime}$ with group $S^{1}$. This base manifold $M^{\prime}$ with positive definite metric $\langle$,$\rangle is called a complex hyperbolic space of holomorphic$ sectional curvature -4 .

In this section, we shall investigate circles in $\mathrm{CH}_{n}(-4)$ by making use of the fibration $\pi: M \rightarrow C H_{n}(-4)$. For the sake of simplicity we identify a vector field $X$ on $C_{n}(-4)$ with its horizontal lift $X^{*}$ on $M$. We denote by $J$ the natural complex structure on $C^{n+1}$. We mix the complex structures of $C^{n+1}$ and $\mathrm{CH}_{n}(-4)$. Let $z$ be a point of $M$. We denote by $N=N(z)$ the position vector of the point $z$. Note that the integral curves of tangent vector field $J N$ are the fibers of the fiber bundle $\pi: M \rightarrow C H_{n}(-4)$. The relation between the connection $\bar{\nabla}$ of $C^{n+1}$ and the connection $\tilde{\nabla}$ of $M$ is as follows:

$$
\tilde{\nabla}_{U} V=\bar{\nabla}_{U} V-\langle U, V\rangle N
$$

for any vector fields $U$ and $V$ on $M$. In fact, since $\langle N, N\rangle=-1,\langle V, N\rangle=0$ and $\bar{\nabla}_{U} N=U$, we get

$$
\begin{aligned}
\tilde{\nabla}_{U} V & =\bar{\nabla}_{U} V-\left(\left\langle\bar{\nabla}_{U} V, N\right\rangle /\langle N, N\rangle\right) N \\
& =\bar{\nabla}_{U} V+\left\langle\bar{\nabla}_{U} V, N\right\rangle N=\bar{\nabla}_{U} V-\langle U, V\rangle N .
\end{aligned}
$$

Let $\nabla$ denote the Riemannian connection of $\mathrm{CH}_{n}(-4)$. We then find

$$
\nabla_{X} Y=\tilde{\nabla}_{X} Y+\langle X, J Y\rangle J N
$$

for any vector fields $X$ and $Y$ on $C_{n}(-4)$. In fact, since $\langle J N, J N\rangle=-1$ and $\langle Y, N\rangle=\langle Y, J N\rangle=0$, we have

$$
\begin{aligned}
\nabla_{X} Y & =\tilde{\nabla}_{X} Y-\left(\left\langle\tilde{\nabla}_{X} Y, J N\right\rangle /\langle J N, J N\rangle\right) J N=\tilde{\nabla}_{X} Y+\left\langle\tilde{\nabla}_{X} Y, J N\right\rangle J N \\
& =\tilde{\nabla}_{X} Y-\left\langle Y, \bar{\nabla}_{X}(J N)\right\rangle J N=\tilde{\nabla}_{X} Y-\langle Y, J X\rangle J N .
\end{aligned}
$$

Using this relationship we obtain the following fundamental result on circles in a complex hyperbolic space.

Proposition 1. Let $\gamma$ be a circle in $\mathrm{CH}_{n}(-4)$ with curvature $k$ and complex torsion $\tau$ satisfying: $\nabla_{s} X_{s}=k Y_{s}$ and $\nabla_{s} Y_{s}=-k X_{s}$. Then a horizontal lift $\tilde{\gamma}$ of $\gamma$ 
into $M$ is a helix of order 2, 3 or 5 corresponding to $\tau=0, \tau= \pm 1$ and $\tau \neq 0$, \pm 1 . It satisfies the following differential equations:

$$
\left\{\begin{array}{c}
\tilde{\nabla}_{s} X_{s}=k Y_{s} \\
\tilde{\nabla}_{s} Y_{s}=-k X_{s}-\tau J N_{s} \\
\tilde{\nabla}_{s} J N_{s}=-\tau Y_{s}+\sqrt{1-\tau^{2}} Z_{s} \\
\tilde{\nabla}_{s} Z_{s}=\sqrt{1-\tau^{2}} J N_{s}+k W_{s} \\
\tilde{\nabla}_{s} W_{s}=-k Z_{s}
\end{array}\right.
$$

where the unit horizontal vector fields $Z_{s}$ and $W_{s}$ are given by

$$
Z_{s}=1 / \sqrt{1-\tau^{2}} \cdot\left(J X_{s}+\tau Y_{s}\right) \text { and } W_{s}=1 / \sqrt{1-\tau^{2}} \cdot\left(J Y_{s}-\tau X_{s}\right) .
$$

Here we should note that the coefficient matrix of the equation of helix is not skew-symmetric, because $\left\langle J N_{s}, J N_{s}\right\rangle=-1$.

We show Theorem 1 by dividing into three cases; $\tau=0, \tau= \pm 1$ and otherwise.

Proposition 2. Let $\gamma$ be circle with curvature $k$ on $\mathrm{CH}_{n}(-4)$. Suppose that the complex torsion $\tau$ of $\gamma$ is 0 . Then the following hold:

(i) When $k>1, \gamma$ is a simple closed curve with prime period $2 \pi / \sqrt{k^{2}-1}$.

(ii) When $k \leq 1, \gamma$ is a simple two-sides unbounded open curve.

Proof. By hypothesis a horizontal lift $\tilde{\gamma}$ of $\gamma$ on the hypersurface $M$ satisfies $\tilde{\nabla}_{s} X_{s}=k Y_{s}$ and $\tilde{\nabla}_{s} Y_{s}=-k X_{s}$. We shall solve this differential equation with the initial condition $\tilde{\gamma}(0)=z, X_{0}=u$ and $Y_{0}=v$. By using (1.1) we can rewrite this equation into $\tilde{\gamma}^{(3)}+\left(k^{2}-1\right) \dot{\tilde{\gamma}}=0$ as a equation in $\boldsymbol{C}^{n+1}$. On the other hand since the initial condition is rewritten as $\tilde{\gamma}(0)=z, \dot{\tilde{\gamma}}(0)=u$ and $\ddot{\tilde{\gamma}}(0)=k v+z$, we get

$$
\begin{aligned}
\tilde{\gamma}(s)= & \frac{1}{1-k^{2}}\left(-k^{2}+\cosh \sqrt{1-k^{2}} s\right) \cdot z+\frac{1}{\sqrt{1-k^{2}}} \sinh \sqrt{1-k^{2}} s \cdot u \\
& +\frac{k}{1-k^{2}}\left(-1+\cosh \sqrt{1-k^{2}} s\right) \cdot v, \quad \text { if } k<1, \\
\tilde{\gamma}(s)= & \left(2+s^{2}\right) / 2 \cdot z+s u+\left(s^{2} / 2\right) v, \quad \text { if } k=1, \\
\tilde{\gamma}(s)= & \frac{1}{k^{2}-1}\left(k^{2}-\cos \sqrt{k^{2}-1} s\right) \cdot z+\frac{1}{\sqrt{k^{2}-1}} \sin \sqrt{k^{2}-1} s \cdot u \\
& +\frac{k}{k^{2}-1}\left(1-\cos \sqrt{k^{2}-1} s\right) \cdot v, \quad \text { if } k>1 .
\end{aligned}
$$

These expressions tell us that $\gamma=\pi \circ \tilde{\gamma}$ is two-sides unbounded if $k \leq 1$. 
We now suppose that there exists some $s_{0}$ satisfying $\gamma\left(s_{0}\right)=\gamma(0)$, that is, $\tilde{\gamma}\left(s_{0}\right)=e^{\theta i} \tilde{\gamma}(0)\left(=e^{\theta i} z\right)$ for some $\theta \in[0,2 \pi)$. Since the vectors $z, u$ and $v$ are linearly independent in $C^{n+1}$, these expressions imply the following:

$$
\begin{aligned}
& \left\{\begin{aligned}
\cosh \left(\sqrt{1-k^{2}} s_{0}\right)-k^{2}=\left(1-k^{2}\right) e^{\theta i}, & \\
\sinh \left(\sqrt{1-k^{2}} s_{0}\right)=0, & \text { if } k<1, \\
\cosh \left(\sqrt{1-k^{2}} s_{0}\right)-1=0 . & \text { if } k=1, \\
s_{0}=0, & \text { if } k>1
\end{aligned}\right. \\
& \left\{\begin{aligned}
\cos \left(\sqrt{k^{2}-1} s_{0}\right)-k^{2}=\left(1-k^{2}\right) e^{\theta i}, & \\
\sin \left(\sqrt{k^{2}-1} s_{0}\right)=0, & \\
\cos \left(\sqrt{k^{2}-1} s_{0}\right)-1=0 . &
\end{aligned}\right.
\end{aligned}
$$

This yields that if $k \leq 1$, then $s_{0}=0$, so that $\gamma$ is a simple open curve, and that if $k>1$, then $\sqrt{k^{2}-1} s_{0} / 2 \pi \in Z$ and $\theta=0$. We here note that $\sqrt{k^{2}-1} s_{0} / 2 \pi \in Z$ implies $\dot{\tilde{\gamma}}\left(s_{0}\right)=\dot{\tilde{\gamma}}(0)$ and $\ddot{\tilde{\gamma}}\left(s_{0}\right)=\ddot{\tilde{\gamma}}(0)$, that is, $X_{s_{0}}=u$ and $Y_{s_{0}}=v$. Therefore we can see that $\gamma$ is a simple closed curve with prime period $2 \pi \sqrt{k^{2}-1}$. Of course $\tilde{\gamma}$ is also a simple closed curve with the same prime period.

REMARK. The circle $\gamma$ lies on an embedded real hyperbolic plane $\pi((\boldsymbol{R} z \oplus \boldsymbol{R} u \oplus \boldsymbol{R} v) \cap M)$.

For completeness we investigate the case of $\tau= \pm 1$ (c.f. [1]).

Proposition 3. Let $\gamma$ be a circle with curvature $k$ in $\mathrm{CH}_{n}(-4)$. Suppose that the complex torsion $\tau$ of $\gamma$ is 1 or -1 . Then the following hold:

(i) When $k>2, \gamma$ is a simple closed curve with prime period $2 \pi / \sqrt{k^{2}-4}$.

(ii) When $k \leq 2, \gamma$ is a simple two-sides unbounded open curve.

Proof. We consider the case of $\tau=-1$, that is, $Y_{s}=J X_{s}$. By Proposition 1 , a horizontal lift $\tilde{\gamma}$ of $\gamma$ satisfies the differential equation $\tilde{\nabla}_{s} X_{s}=k J X_{s}$, so that $\ddot{\tilde{\gamma}}-k J \dot{\tilde{\gamma}}-\tilde{\gamma}=0$. Solving this equation under the initial condition $\tilde{\gamma}(0)=z$ and $\dot{\tilde{\gamma}}(0)=u$, we get

$$
\begin{array}{ll}
\tilde{\gamma}(s)=e^{i s}(1-i s) z+s e^{i s} u, & \text { if } k=2, \\
\tilde{\gamma}(s)=\frac{1}{\alpha-\beta}\left(-\beta e^{\alpha s}+\alpha e^{\beta s}\right) z+\frac{1}{\alpha-\beta}\left(e^{\alpha s}-e^{\beta s}\right) u, & \text { if } k \neq 2,
\end{array}
$$


where $\alpha$ and $\beta$ are the solutions of the characteristic equation $t^{2}-$ kit $-1=0$ for the equation for $\tilde{\gamma}$ and satisfy $\alpha+\beta=k i, \alpha \beta=-1$. By the same discussion as in the proof of Proposition 2, we get the conclusion for $\tau=-1$. If we reverse the direction of a circle $\sigma(s)=\gamma(-s)$, then the signature of the complex torsion changes, so that we get our conclusion.

Remark: The circle $\gamma$ lies on $\pi((C z \oplus C u) \cap M)$.

The rest of this section is devoted to the study the case of $\tau \neq 0, \pm 1$. By elementary calculations we get the following two lemmas.

Lemma 1. For fixed $\tau,-1<\tau<1$, the following equation for $k$

$$
k^{2} \tau^{2}-\frac{4}{27}\left(k^{2}-1\right)^{3}=0 .
$$

has a unique positive solution $k(\tau)$, which satisfies the following:

1) $k(\tau) \geq \sqrt{1+(3 / 2)|\tau|} \geq 1$

2) $k(-\tau)=k(\tau)$

3) $k(\tau)$ is a monotone increasing function with respect to $\tau$ when $0 \leq \tau \leq 1$,

4) $k(0)=1$ and $k( \pm 1)=2$.

Lemma 2. We consider the following cubic equation;

$$
\lambda^{3}-\left(k^{2}-1\right) \lambda-k \tau=0,
$$

where $k>0$ and $-1<\tau<1$. Let $k(\tau)$ be the unique positive solution for (1.3). Then the following hold:

(i) When $k<k(\tau)$, the equation (1.4) has two conjugate non-real solutions,

(ii) When $k=k(\tau)$, the equation (1.4) has double root,

(iii) When $k>k(\tau)$, the equation (1.4) has three distinct real solutions.

We are now in a position to prove the following

Proposition 4. Let $\gamma$ be a circle with curvature $k$ and with complex torsion $\tau$ in $\mathrm{CH}_{n}(-4)$. Suppose that $\tau \neq 0, \pm 1$. Let $k(\tau)$ be the unique positive solution for (1.3). Then the following hold:

(1) When $k \leq k(\tau), \gamma$ is a simple two-sides unbounded open curve.

(2) When $k>k(\tau)$, we denote by $a, b$ and $d(a<b<d)$ nonzero real solutions for (1.4). Then we find the following: 
(i) If one of the three ratios $a / b, b / d$ and $d / a$ is rational, $\gamma$ is a simple closed curve. In addition, the prime period of $\gamma$ is the least common multiple of $2 \pi /(b-a)$ and $2 \pi /(d-a)$.

(ii) If each of the three ratios $a / b, b / d$ and $d / a$ is irrational, $\gamma$ is $a$ simple bounded open curve.

Proof. By the first and the second equalities of Proposition 1, a horizontal lift $\tilde{\gamma}$ of $\gamma$ satisfies $\tilde{\nabla}_{s}\left(\tilde{\nabla}_{s} X_{s}\right)=-k^{2} X_{s}-k \tau J N_{s}$. This, together with (1.1), implies that $\tilde{\gamma}^{(3)}+\left(k^{2}-1\right) \dot{\tilde{\gamma}}+k \tau i \tilde{\gamma}=0$. Its characteristic equation

$$
t^{3}+\left(k^{2}-1\right) t+k \tau i=0
$$

yields the equation (1.4) by setting $t=\lambda i$. Solving the equation on $\tilde{\gamma}$ with initial condition $\tilde{\gamma}(0)=z, \dot{\tilde{\gamma}}(0)=u$ and $\ddot{\tilde{\gamma}}(0)=k v+z$, we get the following expression:

$$
\begin{aligned}
& \tilde{\gamma}(s)=A e^{\alpha s}+B e^{\beta s}+C e^{\delta s}, \quad \text { if } k \neq k(\tau), \\
& \tilde{\gamma}(s)=D e^{\mu s}+E e^{v s}+F s e^{v s}, \quad \text { if } k=k(\tau) .
\end{aligned}
$$

Here $\alpha, \beta$ and $\delta$ are distinct solutions for (1.5) in the case of $k \neq k(\tau)$, which satisfy $\alpha+\beta+\delta=0, \alpha \beta+\beta \delta+\delta \alpha=k^{2}-1$, and $\alpha \beta \delta=-k \tau i$. By Lemma 2, when $k>k(\tau)$ they are pure imaginary; $\alpha=a i, \beta=b i, \delta=d i$, and when $k<k(\tau)$ they are expressed as $\alpha=a i, \beta=h+i g, \delta=-h+i g$ with real $a, h, g(h>0)$. When $k=k(\tau)$, the pure imaginary $\mu=a i$ and $v=b i$ are the solutions for (1.5) with $a=-2 b$ and $b=-\operatorname{sgn}(\tau) \sqrt{\left(k(\tau)^{2}-1\right) / 3}$, where $\operatorname{sgn}(\tau)$ denotes the signature of $\tau$. The elements $A, B, C, D, E$ and $F$ of $C^{n+1}$ are given by

$$
\begin{aligned}
& A=\frac{-(\beta \delta+1) z-\alpha u-k v}{(\alpha-\beta)(\delta-\alpha)}, \quad B=\frac{-(\alpha \delta+1) z-\beta u-k v}{(\alpha-\beta)(\beta-\delta)} \\
& C=\frac{-(\alpha \beta+1) z-\delta u-k v}{(\beta-\delta)(\delta-\alpha)}, \quad D=\left\{\left(v^{2}+1\right) z-2 v u+k v\right\} / 9 v^{2} \\
& E=\left\{\left(8 v^{2}-1\right) z+2 v u-k v\right\} / 9 v^{2}, \quad F=\left\{\left(1-2 v^{2}\right) z+v u+k v\right\} / 3 v .
\end{aligned}
$$

By these expressions we can conclude $\gamma$ is two-sides unbounded if $k \leq k(\tau)$, and bounded if $k>k(\tau)$.

We now suppose that there exists some $s_{0}$ satisfying $\gamma\left(s_{0}\right)=\gamma(0)$, that is $\tilde{\gamma}\left(s_{0}\right)=e^{\theta i} \tilde{\gamma}(0)$ for some $\theta \in[0,2 \pi)$. Our discussion consists of the three parts. In the case that $k>k(\tau)$, by the expression of $\tilde{\gamma}$, this condition is equivalent to

$$
\left.A+B \cdot \exp \left((b-a) i s_{0}\right)+C \cdot \exp \left((d-a) i s_{0}\right)\right)=z \cdot \exp \left(\left(\theta-a s_{0}\right) i\right)
$$


Since $\langle z, u\rangle=\langle z, v\rangle=0$ and $|\langle u, v\rangle| \neq 1$, the vectors $z, u$ and $v$ are linearly independent. This equality, with the expression of $A, B$ and $C$, is equivalent to the following:

$$
\left\{\begin{array}{l}
(b-d)(1-b d)+(d-a)(1-d a) \cdot \exp \left((b-a) i s_{0}\right)+(a-b)(1-a b) \\
\quad \times \exp \left((d-a) i s_{0}\right)=(a-b)(b-d)(d-a) \cdot \exp \left(\left(\theta-a s_{0}\right) i\right) . \\
a(b-d)+b(d-a) \cdot \exp \left((b-a) i s_{0}\right)+c(a-b) \cdot \exp \left((d-a) i s_{0}\right)=0 \\
b-d+(d-a) \cdot \exp \left((b-a) i s_{0}\right)+(a-b) \cdot \exp \left((d-a) i s_{0}\right)=0 .
\end{array}\right.
$$

Since $a, b$ and $d$ are different each other, we get $\exp \left((b-d) i s_{0}\right)=1$ from the second and the third equalities of (1.6). Hence we have $(b-d) s_{0} / 2 \pi \in Z$. Similarly we find that $(d-a) s_{0} / 2 \pi \in Z$ and $(b-a) s_{0} / 2 \pi \in Z$. These yield that $(b-a) /(d-a)$ is rational if $s_{0} \neq 0$. As we have $(b-a) /(d-a)=$ $(b+(b+d)) /(d+(b+d))=2-(3 d /(b+2 d))=2-(3 /(2+b / d))$, we get that $b / d$ is rational if $s_{0} \neq 0$. Thus $\gamma\left(s_{0}\right)=\gamma(0)$ implies either $b / d$ is rational or $s_{0}=0$. Therefore if $b / d$ is irrational then the circle is a simple curve. Conversely we suppose that $b / d$ is rational, that is $(b-a) /(d-a)$ is rational. Let $s_{0}$ be the least common multiple of $2 \pi /(d-a)$ and $2 \pi /(b-a)$. Then (1.6) holds with $\theta \equiv a s_{0}(\bmod 2 \pi)$, hence $\tilde{\gamma}\left(s_{0}\right)=\exp \left(a s_{0} i\right) \tilde{\gamma}(0)$. Moreover one can easily get that $\tilde{\gamma}$ satisfies $\dot{\tilde{\gamma}}\left(s_{0}\right)=\exp \left(\right.$ ais $\left.s_{0}\right) \dot{\tilde{\gamma}}(0)$ and $\ddot{\tilde{\gamma}}\left(s_{0}\right)+\tilde{\gamma}\left(s_{0}\right)=\exp ($ ais $) \cdot(\ddot{\tilde{\gamma}}(0)+\tilde{\gamma}(0))$. That is, $X_{s_{0}}=X_{0}$ and $Y_{s_{0}}=Y_{0}$. Therefore we conclude that if $b / d$ is rational then the circle $\gamma$ is a simple closed curve and that its prime period is the least common multiple of $2 \pi /(b-a)$ and $2 \pi /(d-a)$. We here point out that "one of $a / b, b / d$ and $d / a$ is rational" is equivalent to "each of $a / b, b / d$ and $d / a$ is rational". In case that each of $a / b, b / d$ and $d / a$ is irrational, the circle $\gamma$ is a simple open curve.

In the case that $k=k(\tau)$, by the expression of $\tilde{\gamma}$ the condition $\tilde{\gamma}\left(s_{0}\right)=e^{\theta i} \tilde{\gamma}(0)$ leads us to

$$
D \cdot \exp \left(-3 b i s_{0}\right)+E+F s_{0}=z \cdot \exp \left(\left(\theta-a s_{0}\right) i\right)
$$

Since $z, u$ and $v$ are linearly independent, we obtain with the expression of $D, E$ and $F$ that

$$
\begin{array}{r}
-2 \cdot \exp \left(-3 b i s_{0}\right)+2+3 b i s_{0}=0 \\
\exp \left(-3 b i s_{0}\right)-1+3 b i s_{0}=0
\end{array}
$$

Hence $s_{0}=0$, so that the circle $\gamma$ is a simple open curve. 
In the case $k<k(\tau)$, the condition $\tilde{\gamma}\left(s_{0}\right)=e^{\theta i} \tilde{\gamma}(0)$ yields

$$
A+B \cdot \exp \left(h s_{0}+(g-a) i s_{0}\right)+C \cdot \exp \left(-h s_{0}+(g-a) i s_{0}\right)=\exp \left(\left(\theta-a s_{0}\right) i\right) z .
$$

Since $z, u$ and $v$ are linearly independent we get by use of the expression of $A, B$ and $C$ that

$$
\begin{array}{r}
\alpha(\beta-\delta)+\beta(\delta-\alpha) \exp \left(h s_{0}+(g-a) i s_{0}\right)+\delta(\alpha-\beta) \exp \left(-h s_{0}+(g-a) i s_{0}\right)=0 . \\
\beta-\delta+(\delta-\alpha) \exp \left(h s_{0}+(g-a) i s_{0}\right)+(\alpha-\beta) \exp \left(-h s_{0}+(g-a) i s_{0}\right)=0 .
\end{array}
$$

These lead us to

$$
\exp \left(h s_{0}+(g-a) i s_{0}\right)=\exp \left(-h s_{0}+(g-a) i s_{0}\right),
$$

which, together with $h \neq 0$, implies $s_{0}=0$, so the circle $\gamma$ is a simple open curve. Hence we complete the proof.

REMARK. The circle $\gamma$ lies on $\pi((C z \oplus C u \oplus C v) \cap M)$.

Summarizing Propositions 2, 3 and 4 we get Theorem 1. We should note that for given $k>\sqrt{c} k(\tau) / 2$ there exists open circles and closed circles with curvature $k$. We hope the reader compares the result on circles on a complex projective space $([3])$.

\section{Asymptotic behaviours of unbounded circles}

In this section we study the asymptotic behaviours of unbounded circles and show Theorem 2. For this sake the following representation of a complex hyperbolic space is convenient. We can identify $\mathrm{CH}_{n}$ with the open unit ball $D_{n}(C)=\left\{w \in C^{n} \mid \sum_{j=1}^{n} w_{j} \bar{w}_{j}<1\right\}$ in $C^{n}$ by the mapping $\Phi$ defined by $\Phi\left(\pi\left(z_{0}, z_{1}, \ldots, z_{n}\right)\right)=\left(z_{1} / z_{0}, \ldots, z_{n} / z_{0}\right)$. The compactification of a complex hyperbolic space with its ideal boundary is nothing but taking the topological closure of $D_{n}(C)$ with respect to the canonical topology of $C^{n}$.

In the first place we show that a circle $\gamma$ with curvature $k=k(\tau)$ and complex torsion $\tau$ is horocyclic. The circle $\gamma$ with $\gamma(0)=z, \dot{\gamma}(0)=u$ and $\left(\nabla_{s} \dot{\gamma}(s)\right)_{s=0}=k v$ is expressed as

$$
\begin{aligned}
& \gamma(s)=\pi\left(\left(2+s^{2}\right) / 2 \cdot z+s u+\left(s^{2} / 2\right) v\right), \quad \text { if } \tau=0, \\
& \gamma(s)=\pi\left(e^{ \pm i s}(1 \mp i s) z+s e^{ \pm i s} u\right), \quad \text { if } \tau= \pm 1,
\end{aligned}
$$




$$
\begin{aligned}
\gamma(s)= & \pi\left(\frac { - 1 } { 9 b ^ { 2 } } \left[\left\{\left(1-b^{2}\right) e^{-2 b i s}-\left(1+8 b^{2}\right) e^{b i s}+3 b i\left(2 b^{2}+1\right) s e^{b i s}\right\} z\right.\right. \\
& +\left\{-2 b e^{-2 b i s}+\left(2 b+3 b^{2} i s\right) e^{b i s}\right\} J u \\
& \left.\left.+k\left\{e^{-2 b i s}+(-1+3 b i s) e^{b i s}\right\} v\right]\right), \quad \text { if } \tau \neq 0, \pm 1
\end{aligned}
$$

where $b=-\operatorname{sgn}(\tau) \sqrt{\left(k(\tau)^{2}-1\right) / 3}$, which is nonzero when $\tau \neq 0$. We therefore get that $\gamma$ has a single point at infinity;

$$
(\Phi \circ \gamma)(\infty)=\Phi \circ \gamma(-\infty)=\left(\frac{\left(2 b^{2}+1\right) z_{j}+b i u_{j}+k v_{j}}{\left(2 b^{2}+1\right) z_{0}+b i u_{0}+k v_{0}}\right)_{1 \leq j \leq n} .
$$

for any $\tau$. We now show that every geodesic $\rho$ going to the point $(\Phi \circ \gamma)(\infty)$ crosses $\gamma$ orthogonally. One can easily find that the geodesic $\rho$ in $\boldsymbol{C H}_{n}$ which is parametrized by its arc length satisfying $\rho(0)=z$ and $\dot{\rho}(0)=w$ is expressed as:

$$
\rho(s)=\pi(z \cdot \cosh s+w \cdot \sinh s) .
$$

Then

$$
(\Phi \circ \rho)(\infty)=\left(\frac{z_{j}+w_{j}}{z_{0}+w_{0}}\right)_{1 \leq j \leq n} .
$$

The condition $(\Phi \circ \rho)(\infty)=(\Phi \circ \gamma)(\infty)$, together with $(2.1)$, yields that $w=$ $(b J u+k(\tau) v) /\left(2 b^{2}+1\right)$. Since $\quad b=-\operatorname{sgn}(\tau) \sqrt{\left(k(\tau)^{2}-1\right) / 3}$ and $k(\tau) \cdot \tau=$ $b^{3}-\left(k(\tau)^{2}-1\right) b($ recall $(1.4))$, we have

$$
\|b J u+k(\tau) v\|^{2}=b^{2}-2 b k(\tau) \cdot \tau+k(\tau)^{2}=\left(2 b^{2}+1\right)^{2},
$$

so that $w$ is a unit vector. Obviously $\langle w, u\rangle=0$, which guarantees that every geodesic $\rho$ going to the point $(\Phi \circ \gamma)(\infty)$ crosses $\gamma$ orthogonally.

We here interpret $k(\tau)$ in terms of sectional curvatures.

Proposition 5. Let $\gamma$ be a circle with curvarure $k$, complex torsion $\tau(-1 \leq \tau \leq 1)$ and with the associated initial vectors $u, v$. Then $k(\tau)^{2}=$ $|\operatorname{Riem}(u, w)|$, where $w=(b J u+k(\tau) v) /\left(2 b^{2}+1\right), b=-\operatorname{sgn}(\tau) \sqrt{\left(k(\tau)^{2}-1\right) / 3}$ and $\operatorname{Riem}(u, w)$ is the sectional curvature of a plane spanned by $u, w$.

Proof. The curvature tensor $R$ of $\mathrm{CH}_{n}(-4)$ is as follows: $R(X, Y) Z=-(\langle Y, Z\rangle X-\langle X, Z\rangle Y+\langle J Y, Z\rangle J X-\langle J X, Z\rangle J Y+2\langle X, J Y\rangle J Z)$ 
for all vector fields $X, Y$ and $Z$. Hence we have

$$
\operatorname{Riem}(u, w)=-4 b^{2}+8 b k(\tau) \tau-k(\tau)^{2}\left(1+3 \tau^{2}\right)
$$

which, together with $k(\tau) \cdot \tau=b^{3}-\left(k(\tau)^{2}-1\right) b$, yields the conclusion.

In the second place we show that a circle $\gamma$ with curvature $k<k(\tau)$ and complex torsion $\tau$ has two distinct points at infinity. The circle $\gamma$ with $\gamma(0)=z, \dot{\gamma}(0)=u$ and $\left(\nabla_{s} \dot{\gamma}(s)\right)_{s=0}=k v$ is expressed corresponding to $\tau$ as:

$$
\begin{aligned}
\gamma(s)= & \pi\left(\frac{1}{1-k^{2}}\left(-k^{2}+\cosh \sqrt{1-k^{2}} s\right) \cdot z+\frac{1}{\sqrt{1-k^{2}}} \sinh \sqrt{1-k^{2}} s \cdot u\right. \\
& \left.+\frac{k}{1-k^{2}}\left(-1+\cosh \sqrt{1-k^{2}} s\right) \cdot v\right), \quad \text { if } \tau=0 \text { and } k<1 \\
\gamma(s)= & \pi\left(\frac { \operatorname { e x p } ( \pm k i s ) } { \sqrt { 4 - k ^ { 2 } } } \left\{\left(k i \cdot \sinh \sqrt{4-k^{2}} s+\sqrt{4-k^{2}} \cosh \sqrt{4-k^{2}} s\right) z\right.\right. \\
& \left.\left.+\sinh \sqrt{4-k^{2}} s \cdot u\right\}\right), \quad \text { if } \tau= \pm 1 \text { and } k<2, \\
\gamma(s)= & \gamma\left(\frac { - 1 } { ( \alpha - \beta ) ( \beta - \delta ) ( \delta - \alpha ) } \left[\left\{(\beta-\delta)(\beta \delta+1) e^{\alpha s}+(\delta-\alpha)(\alpha \delta+1) e^{\beta s}\right.\right.\right. \\
& \left.+(\alpha-\beta)(\alpha \beta+1) e^{\delta s}\right\} z+\left\{\alpha(\beta-\delta) e^{\alpha s}+\beta(\delta-\alpha) e^{\beta s}+\delta(\alpha-\beta)\right\} u \\
& \left.\left.+k\left\{(\beta-\delta) e^{\alpha s}+(\delta-\alpha) e^{\beta s}+(\alpha-\beta) e^{\delta s}\right\} v\right]\right) \\
& \text { if } \tau \neq 0, \pm 1 \text { and } k<k(\tau) .
\end{aligned}
$$

Here $\alpha=a i, \beta=h+i g$ and $\delta=-h+i g(h>0)$ are distinct solutions for the equation (1.5). Now we remark that for any $\tau(-1 \leq \tau \leq 1)$ we have

$$
(\Phi \circ \gamma)(\infty)=\left(\frac{(\alpha \delta+1) z_{j}+\beta u_{j}+k v_{j}}{(\alpha \delta+1) z_{0}+\beta u_{0}+k v_{0}}\right)_{1 \leq j \leq n} .
$$

and

$$
(\Phi \circ \gamma)(-\infty)=\left(\frac{(\alpha \beta+1) z_{j}+\delta u_{j}+k v_{j}}{(\alpha \beta+1) z_{0}+\delta u_{0}+k v_{0}}\right)_{1 \leq j \leq n} .
$$

Of course the numbers $\alpha, \beta$ and $\gamma$ in the above expressions of $(\Phi \circ \gamma)(\infty)$ and $(\Phi \circ \gamma)(-\infty)$ are distinct solutions for $(1.5)$. However, in case that $\tau=0, \pm 1$, 
in particular we set $\alpha, \beta$ and $\delta$ as follows; When $\tau=0, \alpha=0$ and $\beta=-\delta=\sqrt{1-k^{2}}$. When $\tau= \pm 1, \quad \alpha= \pm k i, \quad \beta=\left( \pm k i+\sqrt{4-k^{2}}\right) / 2 \quad$ and $\delta=\left( \pm k i-\sqrt{4-k^{2}}\right) / 2$.

If we suppose that $(\Phi \circ \gamma)(\infty)=(\Phi \circ \gamma)(-\infty)$, then for $1 \leq j \leq n$

$$
\left(1-\alpha^{2}\right)\left(u_{j} z_{0}-z_{j} u_{0}\right)+k \alpha\left(v_{j} z_{0}-z_{j} v_{0}\right)+k\left(u_{j} v_{0}-v_{j} u_{0}\right)=0 .
$$

Multiplying both hand sides of (2.2) by $\bar{z}_{j}$ and summing up with respect to $j$, we obtain from $\langle z, z\rangle=-1$ and $\langle z, u\rangle=\langle\langle z, v\rangle=0$ that

$$
\left(1-\alpha^{2}\right) u_{0}+\alpha k v_{0}=0 .
$$

Similarly multiplying both sides of (2.2) by $\bar{u}_{j}$ or $\bar{v}_{j}$ and summing up, we get with $\langle u, v\rangle=i\langle u, J v\rangle=i \tau$ that

$$
\begin{aligned}
& \left\{\left(1-\alpha^{2}\right) z_{0}+k v_{0}\right\}-k i \tau\left(\alpha z_{0}-u_{0}\right)=0 . \\
& \left\{\left(1-\alpha^{2}\right) z_{0}+k v_{0}\right\} i \tau+k\left(\alpha z_{0}-u_{0}\right)=0 .
\end{aligned}
$$

When $\alpha=0$, that is $\tau=0$, we have from (2.3), (2.4) and (2.5) that $u_{0}=v_{0}=z_{0}=0$. When $\tau= \pm 1$, that is $v=\mp J u$ and $\alpha= \pm k i$, we find from $(2.4)$ that $z_{0}=0$. But we emphasize that $z_{0} \neq 0$, because $\langle z, z\rangle=-1$. When $\tau \neq 0, \pm 1$, we know that $\alpha \neq 0$. It follows from $[2.4)$ and $[2.5)$ that $\left(1-\alpha^{2}\right) z_{0}+k v_{0}=0$ and $u_{0}=\alpha z_{0}=0$. These imply that

$$
(\alpha \delta+1) z_{0}+\beta u_{0}+k v_{0}=(\alpha \beta+1) z_{0}+\delta u_{0}+k v_{0}=0 .
$$

This, together with the expressions of $(\Phi \circ \gamma)(\infty)$ and $(\Phi \circ \gamma)(-\infty)$, shows that

$$
(\alpha \delta+1) z_{j}+\beta u_{j}+k v_{j}=(\alpha \beta+1) z_{j}+\delta u_{j}+k v_{j}=0 .
$$

Hence $u_{j}=\alpha z_{j}$ for $1 \leq j \leq n$, so that $u=\alpha v$. In any case we have a contradiction. We therefore get $(\Phi \circ \gamma)(\infty) \neq(\Phi \circ \gamma)(-\infty)$, and conclude the assertion of Theorem 2.

\section{References}

[1] T. Adachi, Kähler magnetic flows on a manifold of constant holomorphic sectional curvature, Tokyo J. Math. 18 (1995), 473-483.

[2] T. Adachi, Curvature bound and trajectories for magnetic fields on a Hadamard surface, Tsukuba J. Math. 20 (1996), 225-230.

[ 3 ] T. Adachi, S. Maeda and S. Udagawa, Circles in a complex projective space, Osaka J. Math. 32 (1995), 709-719.

[4] W. Ballmann, M. Gromov and V. Schroeder, Manifolds of nonpositive curvature, Birkhäuser 1985. 
[ 5 ] A. Comtet, On the Landau levels on the hyperbolic plane, Ann. of Physics 173 (1987), 185-209.

[6] S. Maeda and Y. Ohnita, Helical geodesic immersions into complex space forms, Geom. Dedicata 30 (1989), 93-114.

[7] K. Nomizu and K. Yano, On circles and spheres in Riemannian geometry, Math. Ann. 210 (1974), 163-170.

Toshiaki Adachi

Department of Mathematics,

Nagoya Institute of Technology,

Gokiso, Nagoya 466-8555, Japan

e-mail address: d43019a@nucc.cc.nagoya-u.ac.jp

Sadahiro Maeda

Department of Mathematics,

Shimane University

Matsue, Shimane 690, Japan

e-mail address: smaeda@riko.shimane-u.ac.jp 